\title{
Médiévales
}

Langues, Textes, Histoire

57 | automne 2009

Langages politiques, $\mathrm{XII}-\mathrm{XV}^{\mathrm{e}}$ siècle

\section{Qu'est-ce que le peuple? Quelques réfexions sur la littérature politique anglaise à la fin du Moyen Âge}

What Does People Mean? Some Thoughts about the English Political Literature at the End of the Middle Ages

\section{Aude Mairey}

\section{OpenEdition}

\section{Journals}

Édition électronique

URL : https://journals.openedition.org/medievales/5804

DOI : $10.4000 /$ medievales. 5804

ISSN : 1777-5892

\section{Éditeur}

Presses universitaires de Vincennes

Édition imprimée

Date de publication : 20 décembre 2009

Pagination : $53-73$

ISBN : 978-2-84292-241-2

ISSN : 0751-2708

\section{Référence électronique}

Aude Mairey, «Qu'est-ce que le peuple? Quelques réfexions sur la littérature politique anglaise à la fin du Moyen Âge », Médiévales [En ligne], 57 | automne 2009, mis en ligne le 18 janvier 2012, consulté le 22 avril 2022. URL : http://journals.openedition.org/medievales/5804 ; DOI : https://doi.org/10.4000/ medievales.5804 
Médiévales 57, automne 2009, p. 53-74

Aude MAIREY

\section{QU'EST-CE QUE LE PEUPLE? \\ QUELQUES RÉFLEXIONS SUR LA LITTÉRATURE POLITIQUE ANGLAISE DE LA FIN DU MOYEN ÂGE}

$\mathrm{Au}$ cours des $\mathrm{XIV}^{\mathrm{e}}$ et $\mathrm{XV}$ siècles anglais, dans le cadre général de la construction de l'État anglais et de l'élaboration d'un dialogue entre le roi et ses sujets, la structuration et les évolutions de la société politique apparaissent très dynamiques, ce qui est lié, notamment, à la montée en puissance de certains groupes sociaux : la gentry, les hommes de lois, les marchands ${ }^{1}$. Or, ces groupes se veulent aussi les représentants de la communauté anglaise, en particulier dans le cadre du Parlement. À ces mouvements est liée la constitution d'un vocabulaire politique en anglais - un langage commun que tous doivent pouvoir comprendre (et cela dépasse bien sûr largement la dimension politique). Mais la mise en place de ce langage est complexe et non linéaire ${ }^{2}$.

Cela vaut en particulier pour la question des typologies sociales et politiques. Comment chaque groupe se définissait-il et définissait-il les autres ? Les plus hautes strates de la société politique - roi, famille royale et noblesse titrée - sont généralement bien cernées. Mais dès que l'on s'aventure plus bas sur l'échelle sociale, les choses se compliquent, comme en témoignent, par exemple, tous les problèmes de définition de la gentry, en particulier pour ceux qui ne sont pas formellement des chevaliers ${ }^{3}$. Ces problèmes apparaissent comme l'expression d'une hiérarchisation en pleine

1. Pour une synthèse générale, voir en anglais G. HARRISs, Shaping the Nation, England 1360-1461, Oxford, 2005 et en français, J.-P. GENET, La genèse de l'État moderne. Culture et société politique en Angleterre, Paris, 2003.

2. Voir l'ouvrage essentiel de Paul Sтrонм, Politique : Languages of Statecraft between Chaucer and Shakespeare, Notre Dame, Ind., 2005 et notamment son introduction.

3. Sur ce point, je renvoie à l'article que j'ai écrit en collaboration avec Franck CoLLARD, «La société politique en France et en Angleterre au miroir de ses représentations mutuelles », dans France et Angleterre : Deux États, Deux sociétés politiques, J.-P. GENET et J. WatTs dir., à paraître. 
évolution. Dans le même temps se pose la question des catégories politiques : le roi, les élites et (pour schématiser) le peuple. Or, la définition de cette dernière catégorie est très épineuse. Ses fonctions et ses représentations sont souvent présentées de manière ambivalente, avec d'importantes évolutions. $\mathrm{Au}$ cœur du problème se trouve la question des relations entre deux notions clés, celles du peuple et de la communauté ${ }^{4}$. Nous voudrions contribuer à éclaircir les mécanismes de mise en place d'un langage politique commun en nous interrogeant sur l'analyse de la perception du «peuple» par les contemporains.

Pour ce faire, l'étude de la littérature politique anglaise de la période peut se révéler fructueuse. Mais cette littérature est encore trop souvent considérée comme conventionnelle, voire stéréotypée, même par des historiens qui reconnaissent son importance ${ }^{5}$. Il ne faut pas nier la réalité des conventions, mais deux remarques doivent être faites : d'une part, elles constituent un cadre qu'il est important d'appréhender si l'on veut comprendre les représentations sociales et culturelles ainsi que leurs évolutions ; d'autre part, il faut s'attacher aux nuances, aux interstices de liberté présents dans les textes, surtout dans une société où l'on ne conçoit pas les choses en termes de nouveauté et d'innovation ${ }^{6}$. L'étude systématique du vocabulaire utilisé par les auteurs peut constituer en la matière un apport précieux. Bref, la littérature ne doit pas être décontextualisée et, dans cette optique, elle peut nous apprendre beaucoup.

$\mathrm{La}$ 《littérature politique » (avec les guillemets d'usage) anglaise à la fin du Moyen Âge se présente d'abord sous forme poétique, jusque dans les miroirs adressés directement au prince, mais elle recouvre en fait des formes très variées. Cette poésie est de plus en plus composée par des laïcs, même si les clercs ne l'ont pas complètement désertée. Et ces laïcs ne sont pour la plupart pas des nobles, mais des membres de la gentry et/ou des administrateurs, même si leur auditoire a pu être en partie constitué de nobles. Ils représentent donc un segment particulier de la société, pas toujours très proche du centre du pouvoir, mais dont la voix est loin d'être neutre. Parmi ces auteurs, quelques noms majeurs apparaissent, surtout au tournant des XIV ${ }^{\mathrm{e}}$ et $\mathrm{XV}^{\mathrm{e}}$ siècles : Geoffrey Chaucer, mais aussi William Langland, John Gower, ou encore Thomas Hoccleve et John Lydgate. Leurs œuvres ont connu une

4. C'est surtout ce dernier terme qui a fait l'objet d'analyses : $c f$. J. QuiLLET, «Communauté, conseil et représentation», dans Histoire de la pensée politique médiévale, J. H. BuRNs dir., Cambridge, 1988, trad. Paris, 1993, p. 492-539. Le champ de la communauté est aussi au cœur de travaux récents sur l'Angleterre médiévale : voir en particulier l'article fondamental de John Watts sur la question, "Les communes": le sens changeant d'un mot au cours du $\mathrm{XV}^{\mathrm{e}}$ siècle », dans La société politique à la fin $d u X V^{e}$ siècle dans les royaumes ibériques et en France occidentale: élites, peuples, sujets, V. CHALET, H. R. Oliva HerRer, J. VALdeÓN BARUQue et J.-P. GeNeT dir., Valladolid et Paris, 2007, p. 197-216.

5. Voir par exemple HaRriss, Shaping the Nation, op. cit., p. 12-13.

6. Cf. A. MAIREY, Une Angleterre entre rêve et réalité. Littérature et société en Angleterre au XIV siècle, Paris, 2007. 
diffusion importante - souvent plusieurs dizaines de manuscrits ${ }^{7}$. Mais il reste également une foule de poèmes anonymes, généralement assez courts. Parmi eux, beaucoup sont des poèmes de circonstances, écrits pour célébrer (ou dénigrer) un événement, un homme... La plupart ne se retrouvent que dans une ou deux copies, mais leur accumulation même est significative du goût des Anglais pour ce type de littérature.

Pour commencer mon exploration, j'ai retenu sept textes ou ensembles de textes, s'étendant de la fin du XIV siècle à la fin du $\mathrm{XV}^{\mathrm{e}}$ siècle :

- William Langland, Piers Plowman, version B, vers $1380^{8}$.

- John Gower :

Prologue et livre VII de la Confessio amantis, 1390-1393 ${ }^{9}$.

To King Henry IV in praise of peace ${ }^{10}$.

- « Poèmes de la tradition ${ }^{11}$ :

Richard the Redeless, vers 1399-1400 ;

Mum and the Sothsegger, vers 1409.

The Crowned King: on the art of governing (1415).

- Thomas Hoccleve :

The Regement of Princes, vers $1412^{12}$.

Balade to my gracious Lord of York (1411) et To the duke of Bedford (1411)

Richard II interred in Westminster (1413)

Deux ballades à Henry V (1413)

The remonstrance against Oldcastle (1415)

Ballade to Henry V: «Victorious kyng... » (1416) ${ }^{13}$

- Poèmes du manuscrit Digby 102 (Oxford, Bodleian Library), vers 1400-1430 ${ }^{14}$ : Loue, God and Drede, 168 vers (1400)

Mede and muche thank, 88 vers (1400?)

Treuth, reste and pes, 168 vers (1401)

Lerne say wele, say litel, or say noght, 248 vers (1404?)

Wyt and wille, 72 vers

To lyve bodyly is perylous, 72 vers

7. Pour une introduction générale, voir The Cambridge History of Medieval English Literature, D. WALlaCE dir., Cambridge, 1999. Pour une approche très problématisée de cette littérature, voir J. SimpSON, Reform and Cultural Revolution, 1350-1547, Oxford, 2002.

8. William Langland, The vision of Piers Plowman, Version B, A. V. C. Schmidt éd., Londres, 1978, 1987 ; Pierre le laboureur, trad. A. MAIREY, Paris, 1999.

9. John Gower, Confessio Amantis, R. PeCK éd., Kalamazoo, 2000 (en ligne à l'adresse suivante : http://www.lib.rochester.edu/camelot/teams/rpcalint.htm).

10. John Gower, In Praise of Peace, K. Forni éd., Kalamazoo, 2005 (en ligne à l'adresse suivante : http://www.lib.rochester.edu/camelot/teams/forwisint.htm) ; M. LIVINGSTONE éd., Kalamazoo, 2005 (en ligne à l'adresse suivante: http://www.lib.rochester.edu/camelot/ teams/ryppintro.htm).

11. The Piers Plowman Tradition, H. BARR éd., Londres, 1993.

12. Thomas Hoccleve, The Regement of Prince, C. R. Blyth éd., Kalamazoo, 1999 (en ligne à l'adresse suivante : http://www.lib.rochester.edu/camelot/teams/hoccint.htm).

13. Selections from Hoccleve, M. C. SEYMour éd., Oxford, 1981.

14. Twenty-Six Political and Other Poems (Digby 102), J. KaIL éd., Londres, 1904 (Early English Texts Society, o.s. 124). 
Man, knowe thyself and lerne to dye, 120 vers

A good makynge of your delay, 104 vers

With god of love and pes, ye trete, 192 vers (1410)

A good stirring to hevenward, 210 vers

God and man ben made at one, 120 vers (1412)

God save the kyng and kepe the crown, 152 vers (1413)

Mede is worchyng, 168 vers (1414)

Man, be warre, er the be woo, 112 vers (1418)

The descryvyng of mannes membres, 152 vers

A remembraunce of liv folyes, 126 vers (1419)

Love that god loveth, 200 vers

The declaryng of religion, 192 vers (1421)

- Georges Ashby, The Active Policy of a Prince, vers $1470{ }^{15}$.

- Poèmes yorkistes ${ }^{16}$ :

Prelude to the wars (1449) et Advice to Court I et II (1450).

Take Good heed, Balat set upponne the yates of Caunterbury, The Battle of Northampton (1460), The battle of Towton (1461), Twelve letters save England (1461).

Edwardus Dei Gracia (1461 ?), A Political retrospect (1462), et The Battle of Barnet (1471).

The death of Edward IV (1483) et The Lily White Rose (1486).

Ce corpus tient son unité du fait qu'il est écrit en anglais et que tous les poèmes abordent des sujets touchant à la société et au gouvernement du pays. Les différences sont cependant importantes, tant sur la forme que sur le fond :

- Piers Plowman et les poèmes dits de sa tradition (Richard, Mum et The Crowned Kyng) se distinguent très formellement des autres poèmes car ils sont allitératifs (la répétition de consonnes et/ou de voyelles est utilisée pour donner sa cohérence au vers). Sur le fond, Piers Plowman, de loin le plus important, est d'abord un poème sur le salut de l'homme et de la société ; Richard, Mum et The Crowned Kyng, s'ils sont directement influencés par Piers au point d'être qualifiés de poèmes de sa tradition, sont plus pragmatiques ${ }^{17}$.

- Le livre VII de la Confessio amantis de Gower (accompagné de son prologue) et le Regement of Princes d'Hoccleve sont des miroirs au prince poétiques qui recourent de façon massive à des exempla pour illustrer leur propos (donner des conseils sur le bon gouvernement) ${ }^{18}$. Si le premier n'a

15. Georges Ashby, The Active Policy of a Prince, M. BATESON éd., Londres, 1899 (Early English Texts Society, e.s. 76).

16. Historical Poems of the Fourteenth and Fifteenth Centuries, R. H. RobBins éd., New York, 1959.

17. Sur ces poèmes, voir MAIREY, Une Angleterre entre rêve et réalité, op. cit. ; H. BARR, Signs and Soth: language in the Piers Plowman tradition, Oxford, 1994.

18. Sur ces questions, voir notamment J. FERSTER, Fictions of Advice: The Literature and Politics of Counsel in Late Medieval England, Philadelphie, 1996; L. SCANLon, Narrative, Authority and Power: The Medieval Exemplum and Chaucerian Tradition, Cambridge, 1994. 
pas été commandité, le second a probablement été commandé (ou du moins impulsé) par Henry V juste avant son accession au trône, vers 1411-1412. The Regement of Princes est donc un poème lancastrien, mais qui dépasse de loin le simple poème de circonstance. Le poème de George Ashby est également un miroir au prince; il date de 1470 environ et a été composé pour le fils d'Henry VI (alors en exil); il ne contient pas d'exempla, seulement des conseils directs à l'héritier du royaume (qui ne règnera en fait jamais) dans la période troublée de la guerre civile ${ }^{19}$.

- Les poèmes du Digby 102 sont en partie des poèmes de circonstances, écrits par un seul auteur, peut-être un clerc londonien qui connaissait bien le parlement, sur une période allant de 1400 à 1430 environ. Les poèmes yorkistes enfin, qui datent de la seconde moitié du $\mathrm{Xv}^{\mathrm{e}}$ siècle et ont tous été composés en faveur de la dynastie yorkiste (qui a écarté la dynastie lancastrienne), sont également des poèmes de circonstances, mais ne sont pas tous du même auteur.

L'étude individuelle et linéaire de ces poèmes est, bien entendu, tout à fait indispensable. Mais il est également fructueux d'effectuer des comparaisons approfondies, en s'aidant de méthodes statistiques. Deux méthodes complémentaires permettent deux angles d'approche différents: l'analyse factorielle et l'analyse lexicologique proprement dite. L'analyse factorielle par correspondance permet l'étude comparative d'un corpus de textes et de plusieurs corpus entre eux. Elle s'interroge sur la distribution des mots dans les textes - les proximités et les oppositions repérées n'étant bien sûr que relatives - et permet une représentation graphique ${ }^{20}$. Elle permet de percevoir des phénomènes à des échelles différentes et surtout de travailler sur des corpus importants ${ }^{21}$.

19. Cf. R. J. MeYer-LeE, «Laureates and beggars in Fifteenth-century English Poetry : the case of George Ashby », Speculum, 79/3, 2004, p. 687-726.

20. Le principe de base d'une AFC est l'étude des écarts entre un tableau de données réelles et un tableau de données théoriques ou neutres. Dans ce dernier, la fréquence de chaque mot est proportionnelle à la taille de chaque texte et à l'effectif total de chaque mot. Les écarts bruts qui résultent de ce calcul doivent cependant être pondérés. En effet, il faut se demander s'ils sont significatifs par rapport à la taille des textes et par rapport à l'importance relative des mots dans chaque texte. L'AFC permet, par une série de mise en facteurs de ces écarts, fondés sur des calculs complexes, de dégager les différentes oppositions entre les textes et les mots, et elle permet la lecture, sur un même graphique, des positions des textes et des mots les uns par rapport aux autres. La proximité entre textes et mots est une information de première importance. Le premier facteur représente les oppositions les plus nettes, suivi du deuxième, du troisième... En général, l'analyse n'est plus très significative au-delà du quatrième facteur (sauf exception). À partir de ces calculs, il est possible de construire une représentation graphique. Les graphiques présentent les facteurs deux par deux. Pour différents exemples, voir Mesurer le texte, Histoire \& Mesure, 18, 2003. Pour cette étude, seuls les substantifs, les verbes et les qualificatifs sont conservés.

21. Bien sûr, comme toute méthode, ces différents types d'analyses ne constituent pas une panacée en elles-mêmes et nécessitent des précautions d'emplois importantes, surtout pour un corpus médiéval. Cf. A. MAIREY, Une Angleterre entre rêve et réalité, op. cit., p. 22-24. 
Que peut donc nous apprendre une analyse factorielle réalisée sur ces poèmes ? Pour une étude complète, étant donnée la taille importante de certains de ces textes, le plus simple est de construire l'analyse à partir des 100 premières fréquences. Deux exceptions cependant : étant donné la petite taille du poème d'Ashby et des textes yorkistes, seules les 50 premières fréquences ont été retenues.

Tabl. 1 : La taille des poèmes (en nombre de mots)

\begin{tabular}{|l|r|}
\hline Langland & 75545 \\
Gower & 42786 \\
Allitératifs & 27774 \\
Digby 102 & 18061 \\
Hoccleve & 48591 \\
Ashby & 7253 \\
Yorkistes & 6861 \\
\hline
\end{tabular}

Tabl. 2 : Les principales fréquences des textes

\begin{tabular}{|c|c|c|c|c|c|c|c|}
\hline & Langland & Gower & Allitératif & Hoccleve & Digby 102 & Ashby & Yorkistes \\
\hline 1 & $\operatorname{men}(377)$ & $\operatorname{man}(228)$ & make (83) & $\operatorname{god}(244)$ & $\operatorname{god}(323)$ & $\operatorname{god}(50)$ & $\operatorname{god}(48)$ \\
\hline 2 & quod (340) & kyng (228) & wel (82) & sayen (237) & $\operatorname{man}(156)$ & $\operatorname{good}(38)$ & englond (45) \\
\hline 3 & $\operatorname{god}(295)$ & sayen $(202)$ & $\operatorname{men}(81)$ & $\operatorname{man}(230)$ & love (122) & $\operatorname{man}(32)$ & day (40) \\
\hline 4 & sayen (292) & stonde (179) & sothe (73) & $\operatorname{good}(215)$ & make (119) & wel (31) & rose (31) \\
\hline 5 & make (228) & $\operatorname{men}(152)$ & knowe (60) & wel (189) & drede (68) & thyng (30) & kyng (31) \\
\hline 6 & $\operatorname{man}(208)$ & make (150) & sayen $(58)$ & $\operatorname{men}(180)$ & sayen (62) & grete (27) & grete (25) \\
\hline 7 & come (203) & $\operatorname{god}(146)$ & mum (58) & make (139) & $\operatorname{men}(62)$ & right (25) & men (24) \\
\hline 8 & wel (156) & take (144) & come $(57)$ & ful (139) & $\operatorname{good}(62)$ & estate (23) & tyme (23) \\
\hline 9 & conscience (149) & wel(135) & kyng (56) & kyng (138) & gyve (59) & high (19) & sayen (23) \\
\hline 10 & crist (144) & wise (122) & peple (52) & lord (126) & lawe (58) & lawe (19) & make (23) \\
\hline 11 & telle (142) & thyng $(120)$ & ful (52) & take (112) & wel (57) & $\operatorname{men}(18)$ & ful (22) \\
\hline 12 & $\operatorname{good}(139)$ & $\operatorname{good}(119)$ & telle (47) & herte (102) & take (56) & kepe (18) & edward (22) \\
\hline 13 & love (137) & telle (110) & $\operatorname{god}(46)$ & thyng (98) & lete (55) & folk (18) & trewe $(21)$ \\
\hline 14 & tyme (128) & sette (94) & $\operatorname{good}(44)$ & right (98) & soule (53) & tyme (17) & preie (21) \\
\hline 15 & knowe (128) & pees $(94)$ & counseil (42) & see $(95)$ & dede (51) & take (17) & floure (21) \\
\hline 16 & kyng (127) & fynde (92) & $\operatorname{man}(41)$ & sone (93) & pees $(47)$ & wise (16) & sprede (20) \\
\hline 17 & take (125) & lawe (88) & shewe (38) & grete (85) & wise (43) & rule (14) & see (19) \\
\hline 18 & holy (125) & world (85) & ende (38) & holde (83) & truthe (42) & peple (13) & come (19) \\
\hline 19 & $\operatorname{lif}(123)$ & first (85) & tyme (37) & fader (79) & mede (42) & make (13) & blessid(18) \\
\hline 20 & kynde (122) & right (84) & lawe (37) & love (77) & synne (41) & truthe (12) & bere (17) \\
\hline 21 & right (116) & grete (81) & right (36) & lete (74) & hevene (39) & entent (12) & $\operatorname{good}(16)$ \\
\hline 22 & truthe (115) & come $(80)$ & wille (35) & gyve (72) & trete $(58)$ & trewe (11) & gentil (16) \\
\hline 23 & piers (115) & speke (75) & wordes (34) & pees (71) & werkes (37) & thynges (11) & wel (14) \\
\hline 24 & wille (113) & falle (72) & kepe (34) & knowe (64) & kepe (37) & lord (11) & take (14) \\
\hline 25 & lord (112) & heren (71) & wite (33) & prince (63) & right (36) & honour (11) & langoure (14) \\
\hline 26 & lawe (103) & kynde (68) & kynde (33) & wise (60) & povere $(36)$ & noble (10) & right (13) \\
\hline 27 & grace (102) & cause (67) & helpe (33) & rede $(60)$ & knowe (36) & lyven (10) & putte (13) \\
\hline 28 & lyve (98) & werre (65) & serve (32) & crist (60) & serve (34) & case (10) & fals (13) \\
\hline 29 & reson (97) & tyme (64) & holde (32) & wey (58) & folk (33) & vertuous (9) & sette (12) \\
\hline 30 & soule (93) & pite (64) & sigger (30) & speke (56) & fals (33) & reson $(9)$ & $\operatorname{man}(11)$ \\
\hline
\end{tabular}


De nombreux termes se retrouvent dans au moins quatre textes (mots en gras) alors que les termes spécifiques n'apparaissant que dans un seul texte (mots en italiques) sont plus rares ${ }^{22}$. Ce tableau des fréquences donne déjà des indices comparatifs, mais il est insuffisant car il ne montre pas les articulations entre les textes. Il faut donc passer à l'analyse factorielle proprement dite.

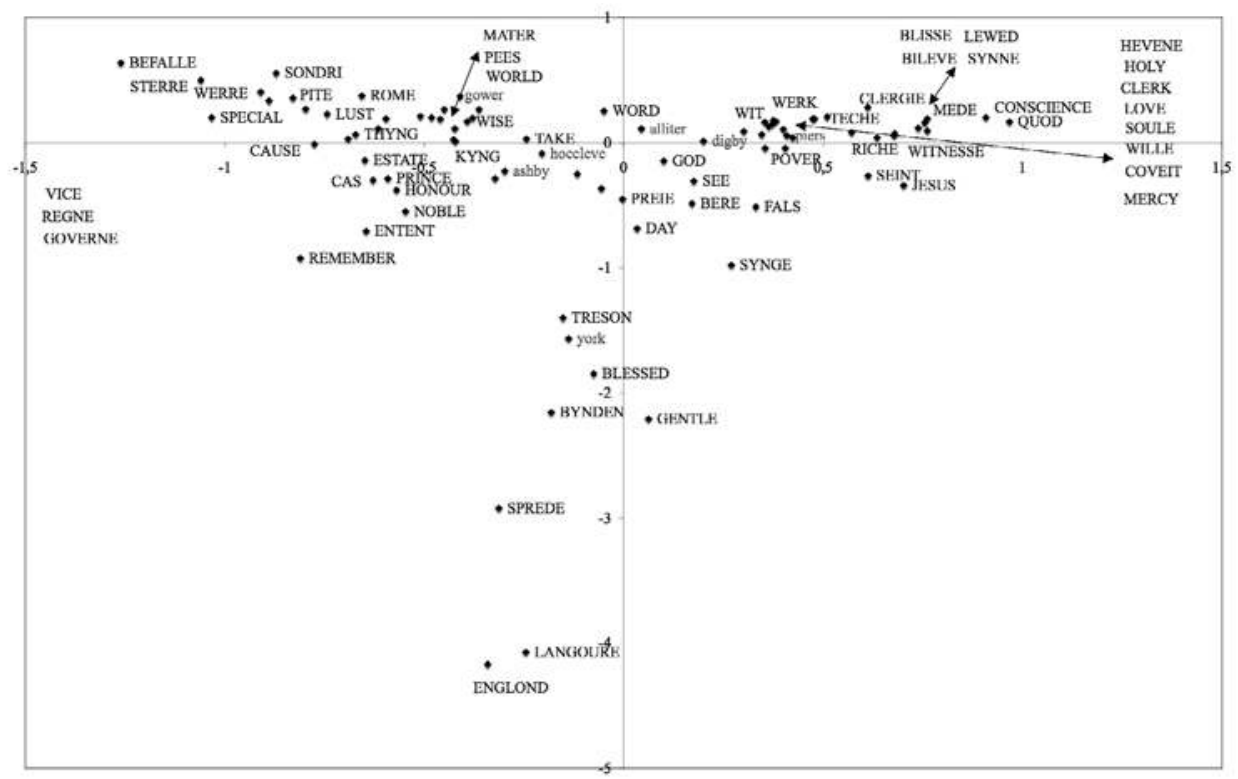

Graph. 1 : L'analyse factorielle générale (facteurs 1 et 2$)^{23}$.

Les facteurs s'échelonnent donc selon l'importance des oppositions entre les textes et les mots. Le premier facteur est le plus important. Il contribue pour près de $30 \%$ à la variance totale, c'est-à-dire presque le tiers des oppositions calculées. Il oppose Piers Plowman et les poèmes du Digby 102 d'un côté à tous les autres textes et en particulier à la Confessio amantis de Gower ${ }^{24}$. Du côté de Piers et des poèmes du Digby, le vocabulaire est de nature surtout spirituelle: bileve, synne, blisse, jesus, clerk, soule... On trouve aussi un vocabulaire de la connaissance et de la communication, sur-

22. Les mots rayés, n'apparaissant que dans un seul texte, n'ont pas été pris en compte dans l'analyse. Par ailleurs, les verbes, substantifs et qualificatifs de même racine ont été regroupés (par exemple : bileve et bileven, counseil et counseillen).

23. Les mots signalés par des flèches sont des mots superposés qui ont été décalés en marge pour qu'ils soient visibles.

24. Les poèmes de la tradition de Piers ne contribuent pas du tout à ce facteur : ils sont pour ainsi dire neutres dans cette opposition. 
tout axé sur la transmission : lernen, techen, witnesse. Enfin, quelques termes spécifiques - c'est-à-dire surreprésentés, voire isolés - apparaissent dans Piers (conscience, quod) ou dans les poèmes du Digby (kyngdom, glosere).

Du côté de Gower et d'Hoccleve, le vocabulaire de la communication et du savoir est également présent, mais il est de nature un peu différente : book, speche, cas, cause, science par exemple. Il s'inscrit de plus aux côtés d'un vocabulaire d'ordre moral ou éthique : pite, justice, vice, vertu... Par ailleurs, le vocabulaire du gouvernement et de la royauté apparaît nettement : kyng en premier lieu, mais aussi regne, governe, prince. Enfin, on trouve de nombreux termes liés à des questions matérielles, et notamment la richesse ou la position dans la société (gold, goodes, take, degre, estate) qui sont plutôt à placer du côté d'Hoccleve et d'Ashby.

L'essentiel ici est donc l'opposition entre d'une part Piers Plowman - et dans une moindre mesure les poèmes du Digby - et d'autre part les miroirs au prince de Gower, Hoccleve et Ashby. Mais il faut également noter la présence importante et la variété du vocabulaire de la communication et de la connaissance dans tous les textes (à l'exception des poèmes yorkistes). Ce sont surtout Langland et Gower qui ont une conception très différente en la matière. Gower s'inspire en grande partie pour son miroir des textes dérivés de la tradition aristotélicienne, ce qui n'est pas le cas de Langland ${ }^{25}$.

Le deuxième facteur contribue pour $22 \%$ à la variance totale, soit près du quart des oppositions. L'opposition principale est très tranchée : elle se situe entre les poèmes yorkistes d'une part, qui sont des poèmes de circonstances employant un vocabulaire assez spécifique, et tous les autres poèmes d'autre part. Les Yorkistes ont un vocabulaire axé sur l'Angleterre (Englond), ainsi que sur la dureté des temps (bynden, langoure, treson...) et la noblesse (gentle). Ils s'occupent davantage des circonstances présentes de la guerre civile, et se soucient moins du bon gouvernement en général (même si certains passages spécifiques peuvent conduire à nuancer ce propos).

Le troisième facteur $(17,8 \%$ de la variance totale, soit un peu moins du cinquième des oppositions repérées) oppose les poèmes du Digby 102 (et dans une bien moindre mesure, le poème d'Ashby) aux autres textes, et en particulier à Piers Plowman. À première vue, cela semble contredire les oppositions dégagées par le premier facteur. En fait, si l'on y regarde de plus près, on s'aperçoit que le vocabulaire spirituel est de nature différente dans chacun de ces deux textes, même si certains termes présents du côté des poèmes du Digby, comme god ou soule, sont aussi très fréquents dans Piers. D'une certaine manière, le vocabulaire des poèmes du Digby est plus basique (hevene, helle), plus combatif aussi (fighte, hate, worship), c'est-àdire plus direct et fait pour convaincre rapidement, le tout, cependant, dans

25. Pour Gower, $c f$. J. Simpson, Science and the self in medieval poetry, Cambridge, 1995 ; pour Langland, voir MAIREY, Une Angleterre entre rêve et réalité, op. cit. 


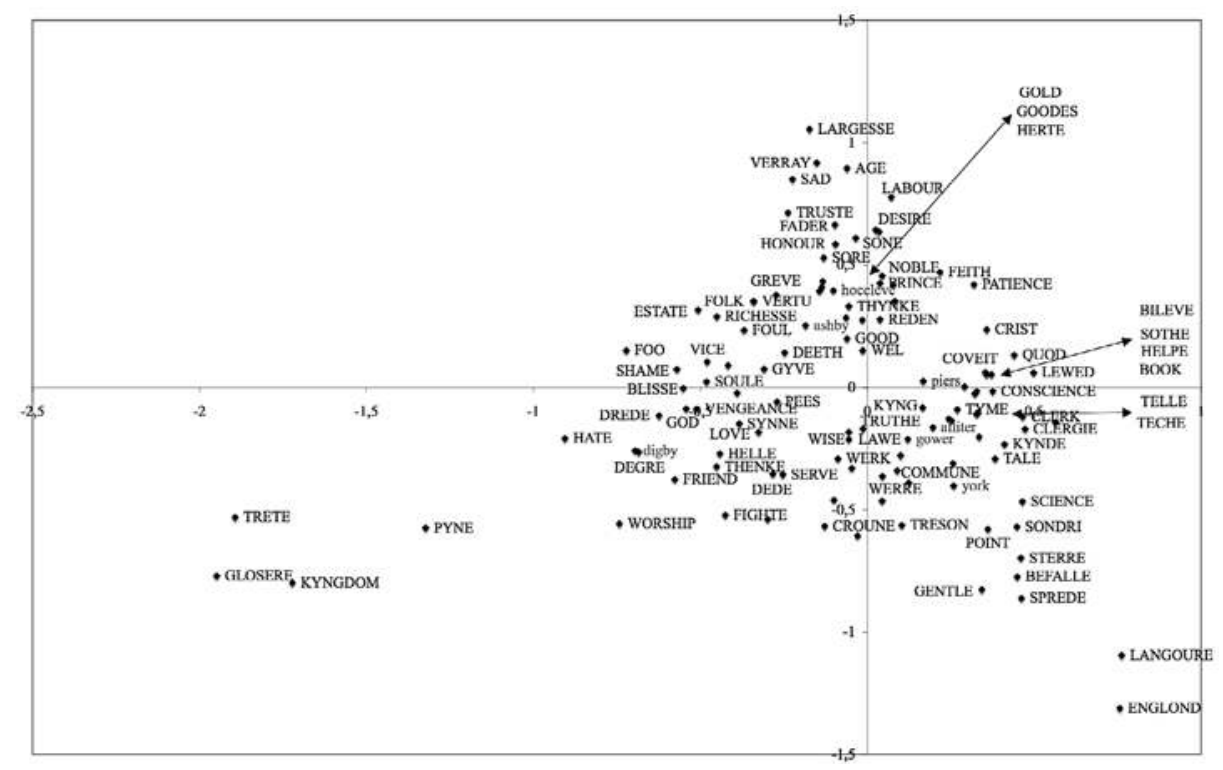

Graph. 2 : L'analyse factorielle générale (facteurs 3 et 4).

le cadre du royaume (kyngdom). À la lecture des textes, on pense d'ailleurs souvent à la littérature sermonnaire. Du côté de Piers et des autres poèmes, on retrouve un vocabulaire abondant sur la communication et le savoir - telle, book, sothe, tale, witnesse - qui renvoie à une réflexion plus approfondie, plus raisonnée aussi, peut-être.

Le quatrième facteur enfin ne contribue plus que pour $12,7 \%$ à la variance. Cette fois, c'est le poème d'Hoccleve (et dans une moindre mesure d'Ashby) qui s'oppose aux autres, à l'exception de Piers Plowman, qui ne participe pas du tout à ce facteur. Les termes associés à Hoccleve sont les plus significatifs: beaucoup relèvent de la situation matérielle et sociale de l'homme (labour, gold, goodes, estate, gyven, largesse), thème cher à Hoccleve. Ce thème se retrouve d'ailleurs dans Ashby. On trouve également chez Hoccleve un vocabulaire lié aux faiblesses de l'homme (sad, sore, desire...) ainsi que la présence de fader et de sone. Tout cela suggère une insistance sur l'homme et sa faiblesse ainsi que l'importance des liens personnels.

Les oppositions entre les poèmes sont donc complexes, mais leur articulation permet de dégager quelques lignes de force. En premier lieu, l'opposition la plus nette se situe entre des textes imprégnés par un vocabulaire de nature spirituelle et les autres. Cela conduit à suggérer que même si le Moyen Âge reste un monde d'abord et profondément chrétien, certains auteurs commencent à envisager d'autres champs de réflexion, de manière plus auto- 
nome $^{26}$. Il faut bien sûr rester nuancé : le poème d'Hoccleve, par exemple, comporte de nombreux passages sur la manière d'être un bon chrétien ${ }^{27}$. En second lieu, le vocabulaire de la communication et de la connaissance est très prégnant, mais il existe de grandes différences de traitement, surtout pour les poèmes de la fin du XIV siècle et de la première moitié du XV siècle ${ }^{28}$. Une différence existe, d'abord entre Gower et Langland, nous l'avons vu, entre une tradition surtout aristotélicienne et une tradition qui est davantage à relier aux aux clercs du XII ${ }^{\mathrm{e}}$ siècle. Mais on note aussi une différence entre les poèmes du Digby 102 et les autres textes, puisque les premiers sont les seuls de la période à ne pas se préoccuper de ces questions - à l'exception de quelques verbes d'enseignement comme lernen ou techen. On le voit, les rapports des auteurs (et de leurs lecteurs) à la communication et à la connaissance sont variés, et moins monolithiques qu'on a pu le dire. Mais surtout, ces domaines sont dans tous les cas intimement liés aux questions sociales et politiques. Enfin, il faut souligner que les oppositions les plus importantes entre ces textes (à l'exception des poèmes yorkistes, un peu à part, troublés par la guerre civile) ne se situent pas tellement dans le domaine du politique. On retrouve pour ce dernier des constantes, même si les mots utilisés varient selon les textes : pour désigner le royaume par exemple, on trouve kyngdom autant que reaume et pour désigner le roi, on trouve prince ou kyng. En effet, s'il existe une opposition entre lexique politique et lexique spirituel dans le premier facteur, celle-ci n'est que partielle et ne se retrouve pas du tout pour les autres facteurs. Il suffit d'ailleurs de regarder le tableau des fréquences maximales pour s'en convaincre: il y a bien un socle commun dans tous les textes autour du roi, du royaume - qui devient cependant de plus en plus l'Angleterre - et de la communauté du royaume, avec les termes peple et commune. Cela ne signifie pas que les poèmes abordent tous les problèmes politiques de la même manière, mais simplement que tous se préoccupent de ces problèmes selon un socle commun qu'il convient de dégager.

Cette analyse générale peut être affinée et complétée par ce que j'appelle une analyse factorielle thématique, construite non plus à partir des fréquences maximales, mais à partir des associations d'un certain nombre de mots appartenant tous au même domaine ${ }^{29}$. Dans le cas présent, il est possible de construire une analyse thématique à partir du vocabulaire des acteurs du gouvernement (au sens le plus large du terme); il s'agit de préciser les remarques précédentes sur le domaine politique. Les termes utilisés pour cette analyse ont été les suivants : kyng/es, prince/s, lordes (au pluriel, car au

26. Sur cette question, voir Sтroнm, Politique, op. cit., p. 16-17.

27. Le narrateur fait par exemple sa profession de foi aux vers 372-392.

28. Les poèmes Yorkistes et Ashby sont exclus de ces observations.

29. Voir A. MAIREY, «Analyses factorielles par domaine lexical: apports et limites. L'exemple de la poésie allitérative anglaise du XIV e siècle », Histoire \& Mesure, 18-3/4, 2003, p. $263-288$. 
singulier le terme renvoie davantage à Dieu), knyght/es, counseil, commune et peple. Certains de ces termes sont problématiques : counseil renvoie autant au conseil proprement dit qu'à l'institution (le conseil royal) ou même simplement aux conseillers en général ; commune soulève également un certain nombre de problèmes qui seront développés plus loin.

Pour garder une certaine cohérence statistique, les listes ont été constituées à partir de l'environnement thématique des termes retenus. La recherche de l'environnement thématique d'un terme, selon l'auteur du logiciel qui permet de la réaliser, Étienne Brunet, est « un calcul particulier, puisqu'on ne recherche plus une accointance entre un mot et un texte, mais une relation privilégiée entre les mots eux-mêmes... La procédure ne se réduit pas ici à deux mots confrontés, mais à l'ensemble indéfini de tous les mots qui peuvent se trouver dans l'entourage d'un mot (ou d'un groupe de mots) qu'on définit comme étant le pôle ${ }^{30}$. Le seuil retenu pour cet environnement a été de 300 caractères. Les substantifs, les adjectifs et les vers apparaissant dans les environnements des différents termes ont ensuite été retenus, à l'exception de ceux pour lesquels les fréquences totales (obtenues en additionnant les fréquences de chaque texte du corpus) étaient inférieures à 15 occurrences. Cette méthode a semblé la plus objective pour la constitution d'une liste par domaine, car l'environnement thématique est une opération effectuée automatiquement par le logiciel. En outre, cet environnement est quantitativement identique pour tous les termes, 300 caractères, soit environ trois vers. Quoi qu'il en soit, cette méthode permet d'inclure un certain nombre de mots dans l'analyse, liés à un thème donné mais pas suffisamment fréquents pour apparaître dans l'analyse générale (par exemple debate, answere, parlement). Elle permet donc d'affiner l'analyse pour un thème précis ${ }^{31}$. Seuls les deux premiers facteurs, les plus significatifs, seront développés.

Le premier facteur contribue pour $34 \%$ à la variance, c'est-à-dire le tiers des oppositions repérées. Comme dans l'analyse générale, Piers Plowman s'oppose aux autres poèmes - surtout ceux de Gower, Ashby et Hoccleve ; mais cette fois, les poèmes du Digby 102 sont dans une position neutre, ainsi que les poèmes allitératifs. Du côté de Piers Plowman, on retrouve les termes spécifiques au poème, comme Conscience, et toujours un vocabulaire spirituel abondant (bileve, jesus, trinite). Il y a aussi de très nombreux acteurs du clergé (bishop, frere, clerk, prelate, preest, pope) ainsi que des termes critiques à son égard, comme simonye. De fait, le clergé est bien une partie intégrante de la société politique pour Langland et, dans une moindre mesure, pour les poèmes de la tradition ${ }^{32}$. Enfin, de très nombreux termes liés à

30. E. Brunet, Manuel de référence pour Hyperbase, version 4.0, CNRS, 1999, p. 38.

31. On notera cependant que, graphiquement, les oppositions apparaissent de manière un peu moins nette, ce qui est logique, étant donné que davantage de mots sont pris en compte, aux fréquences moins importantes.

32. MAIREY, Une Angleterre entre rêve et réalité, op. cit. 


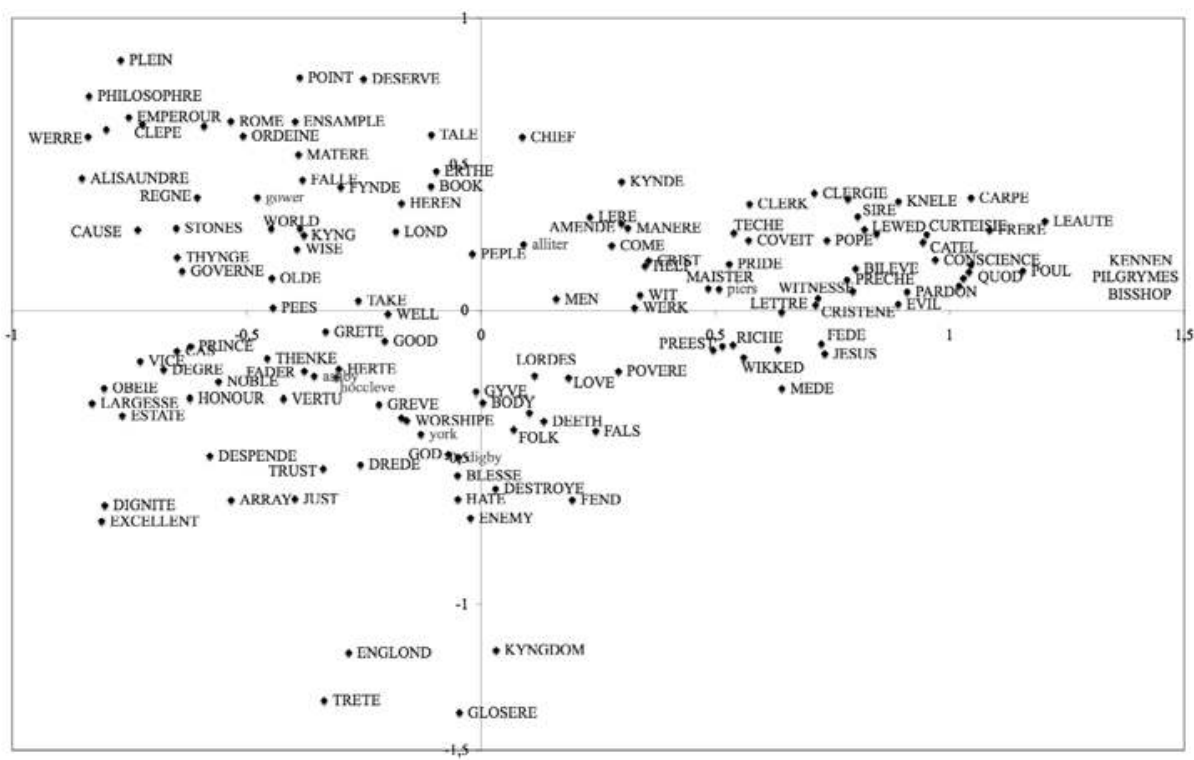

Graph. 3: L'analyse factorielle thématique des acteurs (facteurs 1 et 2).

la transmission et à l'enseignement apparaissent: lere, lernen, construen, prechen; c'est un aspect précis de la communication et du savoir qui apparaît davantage que dans l'analyse générale.

Du côté de Gower et d'Hoccleve, apparaît tout d'abord un vocabulaire relevant très clairement du domaine politique. On y retrouve les principaux acteurs : prince, kyng, royal, emperour... Mais l'essentiel est que ce vocabulaire est très marqué par l'action politique. On trouve notamment des termes généraux comme governe, regne, rule, mysrule; des termes liés à la guerre et à la paix : werre, pees, bataille, vengeance; des termes liés à la conduite des gouvernants envers les gouvernés, notamment dans le cadre de la prospérité du royaume: mayntene, ordeyne, gye, greve, obeie d'une part, profit, welthe, largesse de l'autre. On retrouve par ailleurs un vocabulaire éthique déjà repéré dans l'analyse générale, mais complété par des termes renvoyant à des sentiments (truste, glad, corage...). Enfin, le vocabulaire de la communication et du savoir, toujours présent, est très centré sur le débat, voire la revendication : debate, answere, vois, speken, greve...

Nous avons donc là des oppositions importantes que nous retrouvons quelque peu affinées dans le deuxième facteur, qui ne contribue plus que pour $18 \%$ à la variance totale. Il oppose le texte de Gower, et dans une moindre mesure les poèmes allitératifs, aux poèmes du Digby 102 et aux poèmes yorkistes. Du côté de Gower, il se dégage très nettement un vocabulaire axé sur la communication et la connaissance, plus varié que pour le 
premier facteur : ensample, philosophre, plein, construe, heren, carpen, techen, tale.... Ce vocabulaire se développe dans un cadre politique, avec en particulier plusieurs termes évoquant les rapports des gouvernants et des gouvernés : rule, commande, kyng, mais aussi crye, clayme et parlement. Du côté des poèmes yorkistes et du Digby, le vocabulaire est à nouveau très centré sur l'action, que ce soit dans un contexte troublé (chastise, enemy, hate, destroye, drede, harme, vengeance, rise) ou dans un environnement plus paisible (love, trust, plese, worshipe...). On retrouve là un point commun entre les deux ensembles de textes, que l'on avait pu remarquer séparément dans l'analyse générale, et ce d'autant que, dans les deux cas, l'importance du royaume (kyngdom pour l'un, Englond pour l'autre) est grande.

L'opposition essentielle est toujours celle de Piers Plowman aux autres poèmes, non seulement sur le plan spirituel, mais aussi sur le plan des acteurs du gouvernement et de leurs rapports. La place du clergé dans la société politique est très importante dans Piers Plowman et, de fait, il apparaît en bonne place dans la fondation idéale de la société malgré des critiques nourries. Il faut aussi mettre l'accent sur l'importance de l'enseignement. Un facteur d'explication réside-t-il dans le fait que Langland était un clerc mineur ? C'est possible, mais cela renvoie aussi à la structure même du poème, construite sur une multitude de dialogues entre le narrateur et des figures d'autorité ${ }^{33}$. En tout cas, l'opposition est nette avec les miroirs au prince - ceux de Gower, Ashby et Hoccleve. Dans un cadre éthique toujours présent, ces textes sont plus clairement tournés vers le gouvernement proprement dit et sur les rapports avec les gouvernés. Il faut d'ailleurs remarquer que ces rapports apparaissent surtout à travers les verbes, qui renvoient presque toujours à une action. Ces relations entre gouvernants et gouvernés sont bien sûr inégales : les verbes de commandement et de gouvernement, ou inversement de soumission, dominent largement. Mais cela ne veut pas dire pour autant que les gouvernés n'ont pas voix au chapitre et que les gouvernants n'ont pas de lourdes responsabilités, comme le suggère la présence de termes tournant autour du débat ou de la revendication, dans les miroirs au prince mais aussi dans les poèmes de circonstances, très marqués par l'action.

Il existe donc bien un socle commun entre ces différents poèmes, repéré dans l'analyse générale. Mais dans le détail, des nuances importantes apparaissent. Les stéréotypes et les conventions ne sont pas absents, mais ils peuvent être détournés par chaque auteur pour transmettre ce qui lui semble essentiel dans un cadre donné. L'analyse factorielle permet donc de dégager des structures sous-jacentes qui ne sont pas immédiatement perceptibles dans

33. Voir A. MiddLeton, « Narration and Invention of Experience : Episodic Form in Piers Plowman », dans The Wisdom of Poetry, L. Benson et S. Wenzel dir., Kalamazoo, 1982, p. 81-122. 
une lecture linéaire, même si cette dernière est irremplaçable. Elle peut cependant être utilement complétée par l'analyse lexicologique, dont l'objectif est surtout d'étudier les associations d'un mot donné.

L'analyse lexicologique consiste, comme son nom l'indique, à étudier le lexique de chaque texte. Nous envisagerons ici les concordances et les contextes des mots étudiés ${ }^{34}$. Les mots associés à un terme donné peuvent généralement être divisés en plusieurs groupes : les «personnages » ou groupes de personnage, les notions, les qualificatifs et les verbes. Pour nous interroger plus avant sur la place des gouvernés, nous voudrions nous attacher à l'étude des deux termes les plus fréquents dans les poèmes pour désigner les gouvernés, peple et commune qui, il faut le souligner, ne sont pas apparus dans une opposition particulière au sein de l'analyse factorielle.

Plus encore que le terme français moderne (le terme anglais vient d'ailleurs de l'ancien français et il est surtout utilisé à partir de la seconde moitié du XIV ${ }^{\mathrm{e}}$ siècle), peple revêt, comme le suggère le Middle English Dictionary, un ensemble de significations vastes allant d'un ensemble d'hommes et de femmes à la retenue d'un seigneur en passant par les habitants d'une région, les sujets d'un prince ou la populace - le bas peuple. Peple est présent dans tous les textes, pratiquement toujours dans les 100 premières fréquences et presque toujours au singulier (à l'exception des poèmes du Digby et des Yorkistes). C'est donc un terme courant. L'étendue de ses significations soulève évidemment des difficultés, dans la mesure où tous ces sens peuvent être utilisés par les poètes. Mais même si peple n'apparaît pas toujours dans un sens strictement politique, certains éléments significatifs apparaissent dans l'analyse des associations.

Tabl. 3 : Les associations de peple (tableau simplifié)

\begin{tabular}{|c|c|c|c|c|c|c|c|}
\hline & $\begin{array}{l}\text { Langland } \\
\quad(93)\end{array}$ & $\begin{array}{c}\text { Gower } \\
\text { (61) }\end{array}$ & $\begin{array}{c}\text { Allitér. } \\
\text { (52) }\end{array}$ & $\begin{array}{c}\text { Digby } \\
\text { (14) }\end{array}$ & $\begin{array}{l}\text { Hoccleve } \\
\text { (62) }\end{array}$ & $\begin{array}{c}\text { Ashby } \\
\text { (13) }\end{array}$ & $\begin{array}{c}\text { Yorkistes } \\
\text { (4) }\end{array}$ \\
\hline $\begin{array}{l}\text { Person- } \\
\text { nages }\end{array}$ & $\begin{array}{l}\text { Poul } \mathbf{4} \\
\text { Conscience } \mathbf{2} \\
\text { Christ } \mathbf{2} \\
\text { God } \mathbf{2} \\
\text { Kynde } \mathbf{2} \\
\text { Pees } \mathbf{2} \\
\text { Pilat } \mathbf{2} \\
\\
\text { preest/es } \mathbf{5} \\
\text { clerkes 2 } \\
\text { curatours } \mathbf{2} \\
\text { prisoners } \mathbf{2}\end{array}$ & $\begin{array}{l}\text { God } \mathbf{5} \\
\text { kyng/es } \mathbf{5}\end{array}$ & $\begin{array}{l}\text { kyng } 2 \\
\text { lord } 2 \\
\text { prince/s } 2\end{array}$ & $\begin{array}{l}\text { God } 6 \\
\text { governors } \\
\text { kyng } \\
\text { lordes } \\
\text { riches }\end{array}$ & $\begin{array}{l}\text { kyng } \mathbf{5} \\
\text { lord } \mathbf{2}\end{array}$ & god & $\begin{array}{l}\text { kyng } \\
\text { Jesus }\end{array}$ \\
\hline
\end{tabular}

34. Il s'agit de dégager les termes associés à un mot dans un texte, d'étudier donc l'architecture interne de ce dernier. 


\begin{tabular}{|c|c|c|c|c|c|c|c|}
\hline $\begin{array}{l}\text { Substan- } \\
\text { tifs }\end{array}$ & $\begin{array}{l}\text { pardon } \mathbf{3} \\
\text { pees } \mathbf{3} \\
\text { place/s } \mathbf{3} \\
\text { wordes } \mathbf{3} \\
\text { dede/s } \mathbf{2} \\
\text { good } \mathbf{2} \\
\text { penaunce } \mathbf{2} \\
\text { plentee } \mathbf{2} \\
\text { sighte } \mathbf{2} \\
\text { truthe } \mathbf{2} \\
\text { wombe } \mathbf{2}\end{array}$ & $\begin{array}{l}\text { lond } \mathbf{4} \\
\text { werre } \mathbf{4} \\
\text { Irahel } \mathbf{3} \\
\text { lawe } \mathbf{3} \\
\text { kynghode } \mathbf{2} \\
\text { reste } \mathbf{2} \\
\text { word/es } \mathbf{2}\end{array}$ & $\begin{array}{l}\text { pees } \mathbf{3} \\
\text { contree } \mathbf{2} \\
\text { croune } \mathbf{2} \\
\text { lawe } \mathbf{2} \\
\text { mayntenance } \mathbf{2} \\
\text { multitude } \mathbf{2} \\
\text { penyes } \mathbf{2} \\
\text { peril } \mathbf{2} \\
\text { pleynte/s } \mathbf{2} \\
\text { prece } \mathbf{2} \\
\text { profit } \mathbf{2} \\
\text { synne } \mathbf{2}\end{array}$ & $\begin{array}{l}\text { governance } \mathbf{3} \\
\text { unite } \mathbf{3} \\
\text { degre } \mathbf{2} \\
\text { lawe } \mathbf{2} \\
\text { awe } \\
\text { citee } \\
\text { comandements } \\
\text { debate } \\
\text { estate } \\
\text { lordshipe } \\
\text { right } \\
\text { wille }\end{array}$ & $\begin{array}{l}\text { citee/s } \mathbf{2} \\
\text { justice } \mathbf{2} \\
\text { lond } \mathbf{2} \\
\text { love } \mathbf{2} \\
\text { purs } \mathbf{2} \\
\text { richesse } \mathbf{2} \\
\text { vois } \mathbf{2}\end{array}$ & $\begin{array}{l}\text { herte/s } 2 \\
\text { bille } \\
\text { compassion } \\
\text { entent } \\
\text { favour } \\
\text { highnesse } \\
\text { labour } \\
\text { londe } \\
\text { peine } \\
\text { puissance } \\
\text { support } \\
\text { wele } \\
\text { wilfulnesse }\end{array}$ & $\begin{array}{l}\text { mercy } \\
\text { vois }\end{array}$ \\
\hline Qualific. & $\begin{array}{l}\text { povere 10 } \\
\text { cristene } \mathbf{6} \\
\text { commune } \mathbf{4} \\
\text { lewed } \mathbf{3} \\
\text { bisy } \\
\text { diverse } \\
\text { nedy } \\
\text { plener }\end{array}$ & $\begin{array}{l}\text { commune } 7 \\
\text { litel } \mathbf{2} \\
\text { worthi } 2 \\
\text { desconfit } \\
\text { glade } \\
\text { lewed } \\
\text { liege } \\
\text { menable } \\
\text { mistorned } \\
\text { noble } \\
\text { oppressed } \\
\text { overlein }\end{array}$ & $\begin{array}{l}\text { povere } \mathbf{4} \\
\text { commune } \mathbf{2} \\
\text { coveitous } \mathbf{2} \\
\text { feithles } \\
\text { principal } \\
\text { reccheles } \\
\text { selcouthe } \\
\text { unwise }\end{array}$ & povere & $\begin{array}{l}\text { povere } 4 \\
\text { cheer } \\
\text { commune } \\
\text { glad } \\
\text { hungry } \\
\text { light } \\
\text { nedy } \\
\text { wers }\end{array}$ & $\begin{array}{l}\text { commune } \\
\text { povere } \\
\text { wele }\end{array}$ & $\begin{array}{l}\text { englyssh } \\
\text { trewe }\end{array}$ \\
\hline $\begin{array}{l}\text { Verbes } \\
\text { (comp.) }\end{array}$ & $\begin{array}{l}\text { preche } \mathbf{9} \\
\text { love } \mathbf{3} \\
\text { amende } \mathbf{2} \\
\text { deceyve 2 } \\
\text { helpe 2 } \\
\text { plese 2 } \\
\text { preie 2 } \\
\text { rule 2 } \\
\text { save 2 } \\
\text { teche 2 } \\
\text { telle 2 } \\
\text { wisse 2 }\end{array}$ & $\begin{array}{l}\text { governe } \mathbf{3} \\
\text { lede } 2 \\
\text { pile } 2 \\
\text { take } 2\end{array}$ & $\begin{array}{l}\text { preche } \mathbf{3} \\
\text { lede } \mathbf{2} \\
\text { preve } 2\end{array}$ & $\begin{array}{l}\text { governe } 4 \\
\text { greve } \\
\text { peyne }\end{array}$ & $\begin{array}{l}\text { oppresse } 4 \\
\text { kepe } 2 \\
\text { gye } 2\end{array}$ & $\begin{array}{l}\text { lete } \\
\text { mayntene } \\
\text { surprise }\end{array}$ & \\
\hline
\end{tabular}

On compte 93 occurrences de peple dans Piers. Les associations les plus significatives sont les qualificatifs de peple : povere $\{10\}^{35}$, cristene $\{6\}$, commune et lewed. Langland utilise peple au sens des humbles, des modestes, mais aussi et concomitamment des chrétiens. Pour les personnages associés à ce terme, on note une domination très nette des membres du clergé (preest/es, clerkes, curatours). Les substantifs associés sont variés, souvent liés à l'allitération; un certain nombre de termes relèvent cependant de la dévotion (pardon, penaunce) et des aspects matériels de la vie (good, plentee, wombe).

35. Les chiffres entre accolades renvoient au nombre d'associations avec le terme analysé. 
En ce qui concerne les verbes associés, peple se retrouve peu en position de sujet mais souvent en position de complément. Dans ce cas, prechen $\{9\}$ domine très largement, renforcé par d'autres verbes d'enseignement (techen et wissen) ; plusieurs verbes suggèrent également que le peuple doit être aimé (love) et protégé (kepen, saven), mais aussi réformé (amende); d'autres indiquent également qu'il est régulièrement maltraité (deceyve, mais aussi gile, peynen, poisen). Langland utilise donc surtout peple dans le sens du petit peuple des chrétiens qui doit être guidé et protégé, en particulier par le clergé.

Les autres textes, en revanche, utilisent davantage peple dans un sens politique. Chez Gower (61 occurrences), le peuple est d'abord qualifié de commune $\{7\}$ et il est fortement associé à kyng/es (mais aussi à God). Les substantifs associés les plus fréquents sont lond, werre et lawe. Beaucoup d'autres termes se rattachent au gouvernement et à la communication. Les verbes pour lesquels peple est en position de complément suggèrent également qu'il s'agit des sujets du prince : ils doivent être gouvernés et conduits (governe, lede, guide, rule) tout en étant protégés (kepe, preserve). Enfin, un certain nombre de qualificatifs et de verbes suggère que l'on nuit beaucoup au peuple : mistorned, oppressed, beguile, destroye, greve... (et Gower rejoint ici un peu Langland). Le terme représente donc avant tout les sujets du prince qui doivent être bien gouvernés - mais des sujets enracinés dans un territoire.

Pour les poèmes allitératifs (52 occurrences), on retrouve en association les acteurs dominants de la société politique et les thèmes du gouvernement et de la communication, mais aussi, de manière plus prononcée, des termes concernant la paix, la prospérité et la justice. Il en est de même pour les poèmes du Digby 102 (14 occurrences), avec pour ces derniers une insistance sur le thème du gouvernement; et chez Hoccleve (62 occurrences), avec cette fois une insistance sur le territoire (citee, lond, contree) et la prospérité (purs, richesse mais aussi largesse, moneie, plentee). Mais dans le Regement of Princes, on observe également la présence de plusieurs verbes, dont peple est sujet, qui renvoient à des protestations (curse, grucche, rumble). Cet aspect est spécifique à Hoccleve. Chez Ashby enfin (13 occurrences), ce sont plutôt les sentiments pour le peuple qui constituent la principale préoccupation du poète (herte, compassion, peine).

Commune est un peu moins fréquent que peple et soulève un certain nombre de problèmes dans le sens où il peut être à la fois un substantif et un qualificatif ; or, la distinction n'est pas toujours évidente (qu'en est-il pour commune profit ou commune right ?). Un tableau séparé a donc été réalisé pour les occurrences où commune est en position de qualificatif, sachant que le doute subsiste. Par ailleurs, le substantif commune a des significations multiples, comme le montre l'étendue des définitions dans le Middle 
English Dictionary ou l'Oxford English Dictionary. Il peut signifier la communauté dans son entier, les gens du commun, les Communes du Parlement, mais aussi les biens matériels. S'il y a ambiguïté, on s'aperçoit pourtant que le terme a une forte connotation politique dans tous les poèmes, même s'il ne s'agit pas spécifiquement des Commons en Parlement. En fait, il y a dans les textes un jeu probable sur les différentes acceptions de commune.

Tabl. 4 : Les associations de commune (tableau simplifié)

- Substantif :

\begin{tabular}{|c|c|c|c|c|c|c|c|}
\hline & $\begin{array}{l}\text { Langland } \\
\text { (35) }\end{array}$ & $\begin{array}{c}\text { Gower } \\
\text { (5) }\end{array}$ & $\begin{array}{l}\text { Allitér. } \\
(\mathbf{8})\end{array}$ & $\begin{array}{c}\text { Digby } \\
\text { (17) }\end{array}$ & $\begin{array}{l}\text { Hoccleve } \\
\text { (0) }\end{array}$ & $\begin{array}{c}\text { Ashby } \\
\text { (6) }\end{array}$ & $\begin{array}{c}\text { Yorkistes } \\
\text { (9) }\end{array}$ \\
\hline Personnages & $\begin{array}{l}\text { Conscience } \mathbf{5} \\
\text { Kynde Wit } \mathbf{3} \\
\text { kyng } \mathbf{1 0}\end{array}$ & lord/es 3 & \begin{tabular}{|l|} 
kyng 4 \\
knyght/es 2
\end{tabular} & $\begin{array}{l}\text { lordes } 5 \\
\text { clergy } \mathbf{2}\end{array}$ & & & $\begin{array}{l}\text { kyng } 5 \\
\text { lordes }\end{array}$ \\
\hline Substantifs & $\begin{array}{l}\text { catel } \mathbf{2} \\
\text { court } \mathbf{2} \\
\text { knyghthood } \mathbf{2}\end{array}$ & $\begin{array}{l}\text { accord } \\
\text { duete } \\
\text { exil } \\
\text { love } \\
\text { profit } \\
\text { speche } \\
\text { world }\end{array}$ & $\begin{array}{l}\text { catel } \\
\text { cause } \\
\text { confort } \\
\text { cost } \\
\text { dede } \\
\text { grotz } \\
\text { subsidie }\end{array}$ & kyngdom 2 & $\begin{array}{l}\text { helpe } \\
\text { lordship } \\
\text { ydelnesse }\end{array}$ & $\begin{array}{l}\text { Englond } \\
\text { consent } \\
\text { love } \\
\text { nede } \\
\text { reson }\end{array}$ & \\
\hline Qualificatifs & $\begin{array}{l}\text { carefulle } \\
\text { tresor }\end{array}$ & & & $\begin{array}{l}\text { flour } \\
\text { povere } \\
\text { riche } \\
\text { unkonnyng } \\
\text { witnesse }\end{array}$ & & $\begin{array}{l}\text { povere } 2 \\
\text { welthy } 2\end{array}$ & \\
\hline Verbes (sujet) & \begin{tabular}{|l} 
assente \\
callen \\
clamare \\
coveite \\
crye \\
gyve \\
kepe \\
love \\
knowe \\
quod
\end{tabular} & & $\begin{array}{l}\text { contre } \\
\text { paie } \\
\text { talke }\end{array}$ & $\begin{array}{l}\text { lede } \\
\text { mayntene } \\
\text { rise } \\
\text { robbe } \\
\text { slo }\end{array}$ & & $\begin{array}{l}\text { kepe } 2 \\
\text { lete }\end{array}$ & $\begin{array}{l}\text { drede } 4 \\
\text { helpe } \\
\text { sayen }\end{array}$ \\
\hline Verbes (compl.) & $\begin{array}{l}\text { counseil } 2 \\
\text { sompne } 2\end{array}$ & & aske & $\begin{array}{l}\text { bryngen } \\
\text { greve } \\
\text { mayntene } \\
\text { stonde with } \\
\text { stryf with }\end{array}$ & & & \\
\hline
\end{tabular}


- Qualificatif :

\begin{tabular}{|c|c|c|c|c|c|c|}
\hline $\begin{array}{l}\text { Langland } \\
\text { (17) }\end{array}$ & $\begin{array}{c}\text { Gower } \\
\text { (36) }\end{array}$ & $\begin{array}{c}\text { Allitér. } \\
\text { (8) }\end{array}$ & $\begin{array}{c}\text { Digby } \\
(7)\end{array}$ & $\begin{array}{c}\text { Hoccleve } \\
\text { (10) }\end{array}$ & $\begin{array}{c}\text { Ashby } \\
\text { (3) }\end{array}$ & $\begin{array}{c}\text { Yorkistes } \\
\text { (0) }\end{array}$ \\
\hline $\begin{array}{l}\text { peple } 4 \\
\text { woman/men } 4 \\
\text { profit } 3 \\
\text { court } \\
\text { foode } \\
\text { laborers } \\
\text { lawe } \\
\text { lif } \\
\text { men }\end{array}$ & $\begin{array}{l}\text { peple } \mathbf{7} \\
\text { profit } \mathbf{7} \\
\text { right } \mathbf{5} \\
\text { lawe } \mathbf{3} \\
\text { vois } \mathbf{3} \\
\text { clamour } \mathbf{2} \\
\text { fere } \mathbf{2} \\
\text { good } \mathbf{2} \\
\text { assent } \\
\text { counseil } \\
\text { drede } \\
\text { strif } \\
\text { wronges }\end{array}$ & $\begin{array}{l}\text { peple } 2 \\
\text { clamour } \\
\text { counseil } \\
\text { profit } \\
\text { speche } \\
\text { tale } \\
\text { wele }\end{array}$ & $\begin{array}{l}\text { vois } 3 \\
\text { lawe } 2 \\
\text { noise } \\
\text { profit }\end{array}$ & $\begin{array}{l}\text { profit } 4 \\
\text { cost } \\
\text { goodes } \\
\text { lawe } \\
\text { peple } \\
\text { treson } \\
\text { vois }\end{array}$ & $\begin{array}{l}\text { commune } \\
\text { sawe } \\
\text { wele }\end{array}$ & \\
\hline
\end{tabular}

Dans Piers Plowman, le substantif est fréquent (35 occurrences). Les associations avec kyng dominent $\{10\}$, et l'on retrouve d'autres acteurs de la société politique (clerkes, lordes). Les notions associées sont variées : certains termes expriment la prospérité sous ses diverses formes (catel, profit, welthe) ainsi que le royaume, mais il y a aussi des notions plus générales telles que sothe, truthe, kynde et unkyndenesse. Les verbes montrent que lorsque les commune sont en position de sujet, elles donnent leur assentiment et font des réclamations (assenten, cryen). Lorsqu'elles sont complément d'objet, les verbes concernent le conseil, la protection et le gouvernement (counseillen, rulen, defenden, kepen). Les principaux substantifs qualifiés par commune sont peple et woman, ainsi que profit. Il faut noter que l'expression de commune profit, que l'on traduira en français par bien commun, est importante et renvoie avant tout au bien de la communauté ${ }^{36}$.

Gower utilise peu commune comme substantif (5 occurrences) mais toujours dans un sens politique). En revanche, il l'emploie souvent comme qualificatif (à 36 reprises) pour peple, nous l'avons vu, mais aussi pour profit, right, clamour et vois. Il semble donc que Gower utilise plutôt peple pour évoquer les sujets passifs, alors qu'il emploie des expressions comprenant commune pour exprimer une action plus grande des gouvernés. On retrouve un peu les mêmes thèmes dans les poèmes allitératifs (avec une connotation institutionnelle plus nette dans certains cas), ainsi que dans les poèmes du Digby 102. En revanche, commune n'apparaît pas du tout chez Hoccleve en tant que substantif et assez peu en tant que qualificatif (avec là

36. Sur la notion de bien commun au Moyen Âge, voir M. S. Kempshall, The Common Good in Late Medieval Political Thought, Oxford, 1999. Pour cette notion dans la littérature anglaise, $c f$. A. MAIREY, «Le bien commun dans la littérature anglaise à la fin du Moyen Âge », Revue d'histoire des idées politiques, à paraître. 
encore la domination de profit). Mais on a vu qu'Hoccleve associait à peple des termes relevant de la revendication et de la protestation (dans une optique d'ailleurs assez négative), ce qui n'est pratiquement pas le cas pour les autres.

Ces deux termes - commune et peple - se complètent donc souvent et s'opposent parfois tant au sein du corpus qu'à l'intérieur de chaque poème. Peple est utilisé dans un sens politique dans tous les poèmes - sauf celui de Langland - généralement en lien avec le territoire et la prospérité, mais pas forcément dans le cadre d'un dialogue politique. Chez Gower en particulier, le peuple doit avant tout obéir et se soumettre, même s'il doit être protégé. Chez Hoccleve en revanche, le dialogue apparaît, mais surtout en forme de protestation ${ }^{37}$. Commune en revanche est toujours utilisé dans un sens politique, mais dans une optique de dialogue beaucoup plus nette; Langland est cependant pratiquement le seul à l'employer nettement dans le sens de communauté du royaume. Il me semble donc que l'utilisation à la fois complémentaire et opposée de ces deux termes n'est pas innocente, surtout si elle est replacée dans le contexte de l'existence et de l'affirmation des Communes en parlement. Dans tous les cas (à l'exception d'Hoccleve), le terme commune (à la fois comme substantif et comme qualificatif) est utilisé en rapport avec le fait que les gouvernés - ou au moins une partie d'entre eux - ont la responsabilité de s'exprimer pour le bien du royaume, et cela indépendamment d'un groupe social spécifique, au moins dans un premier temps ${ }^{38}$. Chez Langland, cette dernière apparaît dès le prologue, à travers sa description de la fondation idéale de la société :

Ensuite vint un Roi ; la Chevalerie le précédait ; le pouvoir des Communes l'a établi comme souverain. Alors vint Bon Entendement, qui fit l'ordre des clercs, pour conseiller le Roi et protéger l'intérêt commun. Le Roi, avec la Chevalerie et les clercs, disposa que le peuple assurât leurs moyens de subsistance. Les Communes, par l'action de Bon Entendement, développèrent les compétences et les savoir-faire; et pour le profit de tous désignèrent les laboureurs pour travailler et cultiver la terre en un honnête labeur.

Piers Plowman B, prologue, vers 112-121.

Chez Gower, ce devoir d'expression apparaît également dans le prologue de la Confessio amantis :

Où la loi fait défaut, l'erreur croît. Celui qui n'y croit pas n'est pas sage, car cela a souvent été prouvé auparavant. Ainsi, la clameur commune se trouve dans chaque pays habité, et chacun dit, dans sa complainte, comme le monde

37. Cela doit sans doute être mis en relation avec la position complexe du narrateur face au prince, auquel il demande ses faveurs, tout en s'autorisant à exprimer ses opinions. Sur ces points essentiels, voir N. Perkins, Hoccleve's Regement of Princes : Counsel and Constraint, Cambridge, 2001.

38. Sur cette question, voir aussi E. STEINER, «Commonalty and Literary Form in the 1370s and 1380s », New Medieval Literatures, 6, 2003, p. 199-221. L'auteur étudie notamment la notion de clamour dans différentes sources de la fin du XIV siècle. 
est dans le mal. Et chacun offre à chacun son opinion, d'une étrange manière. Mais quel homme va lui-même considérer sa conscience et ne pas en faire mauvais usage $[\ldots]$ ?

Il n'y a pas de défaut en Dieu, qui se tient toujours dans l'unité. Beaucoup repose donc sur nous, pas seulement sur dix ou douze [personnes], mais sur nous tous, car l'homme est cause de tout ce qui se produira. Pourtant, certains écrivent, et disent, que c'est la fortune qui est à blâmer. Et certains ont pour opinion que c'est une constellation qui est cause de ce qu'un homme fait. Dieu sait bien quelle est la vérité. [...]

Ainsi l'homme est-il pour lui-même complètement la propre cause du bien et du mal.

Confessio amantis, prologue, vers 511-547.

Cependant, ces deux auteurs n'ont pas tout à fait la même conception de la partie des Communes qui peut s'exprimer. Chez Gower, il s'agit assez clairement de la melior pars du royaume. Le peuple proprement dit doit se taire - et certains passages de ses poèmes sont très virulents envers les paysans par exemple ${ }^{39}$. Une tendance similaire apparaît chez les auteurs des poèmes allitératifs qui sont peut-être plus clairs encore dans la mesure où ils font davantage la distinction entre les Communes en parlement d'un côté, et le peuple qui n'a pas voix au chapitre de l'autre. Un passage de Mum and the Sothsegger dénonce d'ailleurs brutalement l'ingérence du commun dans les affaires du royaume :

Vraiment, par le Christ qui m'a créé, je ne peux penser à une cause naturelle pour laquelle les commune devraient contredire la volonté du roi ou interpréter ses actes. Je ne parle pas des chevaliers qui viennent pour les shires, que le roi appelle à son conseil avec d'autres, mais pour ceux qui travaillent, car la loi est contre eux. Pourtant, cela est pratiqué par des gens manquant de sagesse et n'aide en rien, sinon à enflammer les cœurs. Avec leurs histoires, ils détournent l'attention des seigneurs, jusqu'à ce que ces derniers cessent leur travail de défense du pays - s'occuper sur les frontières de battre nos ennemis et maintenir les marches hors du malheur et d'autres choses. Ainsi, les gens du commun bavardent et se nuisent à eux-mêmes, car la conclusion de leur discussion leur nuit fréquemment.

Mum and the Sothsegger, vers 1457-1470.

Langland - et peut-être aussi l'auteur des poèmes du Digby - est plus ambigu. Certes, lui aussi dénonce parfois l'orgueil de certains membres du commun; mais son utilisation de commune - ainsi que de nombreux passages du poème - suggère néanmoins qu'il a peut-être une conception plus large sur la question de savoir qui doit offrir son conseil (sinon participer) au gouvernement.

39. Cf. A. Galloway, "Gower in his most learned role and the Peasant's revolt ", Medievalia, 16, 1992, p. 329-347. 
Dans son article essentiel sur l'évolution de la notion de commune au Moyen Âge, John Watts a émis l'hypothèse d'une hiérarchisation de plus en plus nette du terme, avec une séparation progressive entre les communes en parlement d'une part et les communes en tant que groupe social inférieur d'autre part, par opposition à une relative universalité prévalant auparavant ${ }^{40}$. Cette hiérarchisation peut déjà être entrevue à la fin du XIV e siècle et dans la première moitié $\mathrm{du} \mathrm{XV}^{\mathrm{e}}$ siècle, même si elle reste encore relativement floue. En revanche, il faut insister sur la séparation très nette entre le peuple et les communes, entre les sujets (du roi ou de Dieu) et les membres responsables de la communauté du royaume.

Il n'est pas possible de développer plus avant, dans le cadre de cet article, ces éléments qu'il faudrait mettre plus précisément en relation avec la manière dont les poètes envisagent la royauté, le conseil, la noblesse... Mais ces quelques remarques contribuent, je l'espère, à définir tout autant les fondations d'un langage commun à la société politique anglaise à la fin du Moyen Âge que cette société elle-même - une société politique qui n'est ni unique, ni statique.

Aude MaIrey, CESCM - UMR 6223, 24 rue de la Chaîne - BP 603 F-86022 Poitiers Cedex

Qu'est-ce que le peuple? Quelques réfexions sur la littérature politique anglaise à la fin du Moyen Âge

La société politique anglaise de la fin du Moyen Âge a connu de profondes transformations. La constitution d'un langage politique en anglais, présent notamment dans la littérature contemporaine, en est à la fois un reflet et un moteur. Les incertitudes sur l'expression de catégories-clés, le peuple et la communauté, en témoignent. Leur analyse suggère une séparation accrue entre les sujets du royaume et ceux qui les représentaient, une hiérarchisation croissante, mais avec des hésitations et des tensions.

Littérature politique anglaise - langage politique - peuple - communauté

\section{What Does People Mean ? Some Thoughts about the English Political Literature at the End of the Middle Ages}

At the end of the Middle Ages, english political society knew great transformations. The making of a political language in english, existing notably in contemporary literature, is a reflection as well as a motor of these transformations. The uncertainties about the expression of key categories, people and community, witness this. Their analysis suggests an increased separation between the kingdom's subjects and those who represented them, a society more hierarchised, but with tensions and hesitations.

English political literature - political language - people - community

40. WATTS, «Les “communes" », loc. cit. 
